In addition, anti-TNF treatment starting at early stage (6 weeks) or progressed disease (10 weeks) markedly improved clinical as well as histopathological signs and reversed gait changes.

Conclusions Video-based Catwalk gait analysis is a useful tool for quantitative assessment of functional impairment in inflammatory, destructive arthritis. Joint destruction due to cartilage damage but not synovial inflammation per se is the most important component leading to functional impairment of hTNFtg mice.

\section{A8.8 HYDROGEN SULPHIDE INHIBITS IL-1 $\beta$ STIMULATION OF FIBROBLAST-LIKE SYNOVIOCYTES FROM OSTEOARTHRITIS PATIENTS IN A 3-D MODEL}

doi:10.1136/annrheumdis-2013-203222.8

'D Sieghart, ${ }^{2 H P}$ Kiener, ${ }^{1}, 2 \mathrm{G}$ Steiner, 'B Kloesch. ' $L$ udwig Boltzmann Cluster Rheumatology, Balneology and Rehabilitation, Ludwig Boltzmann Institute of Rheumatology and Balneology, Vienna, Austria; ' $M e d i c a l$ University of Vienna, Department of Internal Medicine III, Division of Rheumatology, Vienna, Austria

Objective Osteoarthritis $(\mathrm{OA})$ is a degenerative and most common joint disease which shows characteristic features like loss of cartilage, formation of osteophytes and alteration of subchondral bone leading to joint impairment and pain. The pathogenesis of $\mathrm{OA}$ is still not fully understood. Fibroblast-like synoviocytes (FLS), occurring in the intimal lining layer of the synovial membrane, were shown to promote secondary synovitis by the release of proinflammatory cytokines and matrix-metalloproteinases (MMPs). The objective of this study was to analyse the possible antiinflammatory potential of hydrogen sulphide $\left(\mathrm{H}_{2} \mathrm{~S}\right)$ on activated FLS cultured in three-dimensional micromass culture.

Methods Primary cell lines based on FLS derived from patients with OA were cultivated in spherical extracellular matrix micromasses. Micromass cultures were stimulated for $1 \mathrm{~h}$ with IL-1 $\beta$ $(10 \mathrm{ng} / \mathrm{ml})$ only or with IL-1 $\beta$ plus either $0.125 \mathrm{mM}$ or $1 \mathrm{mM}$ of sodium hydrogen sulphide ( $\mathrm{NaHS}$ ). As a control, cultures were treated with PBS only. Treatments were applied on day three, five, seven, nine, 12, 14, 16 and 19. Micromasses were cultured for 21 days, fixed with paraformaldehyde, sectioned and stained for hematoxylin and eosin (H\&E), IL-6 or MMP-3. Secretion of IL-6 was analysed by enzyme-linked immunosorbent assay (ELISA).

Results We observed the spontaneous formation of a compacted, lining layer-like architecture by OA-FLS, already described for rheumatoid arthritis (RA)-FLS. Untreated cultures, in addition, showed clusters of elongated cells underneath the condensed cell layer.

A cellular response, which included increased formation of synovial lining as well as changes in cell morphology, could be seen after stimulation with the pro-inflammatory cytokine IL-1 $\beta$. Treatment with $1 \mathrm{mM}$ of $\mathrm{NaHS}$ had the potential to inhibit structural changes caused by cell activation induced by IL-1 $\beta$. Furthermore, $\mathrm{H}_{2} \mathrm{~S}$ treatment reduced the IL-1 $\beta$ stimulation-related elevated levels of IL- 6 secretion.

Conclusions The ability of NaHS to inhibit the development of cellular responses to pro-inflammatory IL- $1 \beta$ could be considered a cartilage protective effect and has to be elucidated in more detail.

\section{A8.9 CHARACTERISATION OF CELL METABOLIC CHANGES IN OSTEOARTHRITIC LABRUM CELLS}

doi:10.1136/annrheumdis-2013-203222.9

'Juchtmans Nele, AM Dhollander Aad, 'Lambrecht Stijn, 'Elewaut Dirk. Department of Rheumatology, Ghent University, Ghent, Belgium

Background and Objectives Little is known about the cell metabolism of the acetabular labrum, a horseshoe-shaped structure sealing the hip joint. It performs similar functions as the menisci in the knee though the metabolic aspects of labrum cells show fibrochondrocyte characteristics, different from menisci and cartilage. The goal of this study was to investigate whether labrum cells derived from osteoarthritic tissue have different cell metabolic characteristics compared with labrum cells derived from healthy tissue. Furthermore this study aimed to investigate specific processes of functional importance in the pathology of osteoarthritis.

Materials and Methods Labrum cells from 5 OA patients en 3 healthy control patients were isolated and cultured in the 3-Dimensional alginate culture system. A genome wide gene expression analysis was performed using the Affymetrix microarray technology. Differential gene expression levels were confirmed on additional patient samples by quantitative PCR (qPCR), western blot and immunohistochemistry.

Results Gene expression analysis showed, as expected, downregulation of aggrecan and several matrix degrading enzymes such as (MMP's) and (ADAMTS's) were up regulated in OA. qPCR confirmation occurred for 20 of the most pronounced differences between normal and OA labrum. Of these 20 genes the Short leucine-rich repeat proteins (SLRP's) asporin (ASPN) and osteomodulin (OMD) were of special interest, both showed a reduced expression level and protein presence in $\mathrm{OA}$ labrum cells. More over stimulation in vitro of labrum cell with IL1 $\beta$ down regulated the expression of both proteins.

Conclusions Labrum cells derived from OA patients show a consistent altered metabolism compared to cells isolated from healthy controls. The reduced expression of ECM genes in OA labrum point towards a distorted ECM synthesis. Together with the observed increase in ECM degradation by MMP's, our data indicate that both distorted anabolism and catabolism contribute to degeneration of labrum tissue in OA. The short leucine-rich proteins (SLPR's) ASPN and $\mathrm{OMD}$ were of special interest as these exhibited the most pronounced expression differences. In conclusion this study clearly indicates that similar events occur during OA development in labrum and cartilage. Though labrum specific expression differences are observed that merit further investigation.

\section{A8.10 MAPK-ACTIVATED PROTEIN KINASE-3 (MK3) REGULATES OSTEOCLAST DIFFERENTIATION AND BONE HOMEOSTASIS}

doi:10.1136/annrheumdis-2013-203222.10

${ }^{1}$ Tobias Braun, 'Gisela Ruiz Heiland, 'Georg Schett, ${ }^{1,2}$ Jochen Zwerina. 'Department of Medicine 3, University of Erlangen-Nuremberg, Erlangen, Germany; ${ }^{2}$ Ludwig Bolzmann Institut of Osteology at the $4^{\text {th }}$ medical Department, Hanusch Hostpital, Vienna, Austria

Background and Objectives MAPK-activated protein kinase-2 (MK2) plays a key role in bone homeostasis. MK2 deficient mice have increased bone mineral density associated with reduced number of osteoclasts. Osteoclast differentiation is impaired due to reduced DNA binding activity of the transcription-factors c-fos and NFATc1. MK3 is besides MK2 an important downstream target of MAPK p38. MK3 plays together with MK2 an important role in regulation of cytokine secretion and inflammation. Aim of our study is to determine the role of $\mathrm{MK} 3$ and $M K 3 / M K 2$ interaction in bone homeostasis.

Materials and Methods We analysed trabecular bone structure of the tibia of 12 week old wild type, MK3 deficient, MK2 deficient and $M K 2 / 3$ deficient mice by micro CT. Tibiae were decalcified and paraffin sections were stained with tartrate-resistant acid phosphatase (TRAP) to determine osteoclast number/bone perimeter and osteoclast surface/bone surface by histomorphometry. Bone marrow cells of wild type, MK3 deficient, MK2 deficient and MK2/3 deficient mice were simulated with M-CSF and RANKL and stained for TRAP after four days to investigate osteoclast differentiation ex vivo.

Results Analysis of trabecular bone structure showed increased trabecular volume, increased trabecular number and decreased 